\title{
Editorial: Developments in Acoustic, Phononic, and Mechanical Materials for Wave Control
}

\author{
Chengzhi Shi ${ }^{1,2 *}$ \\ ${ }^{1}$ George W. Woodruff School of Mechanical Engineering, Georgia Institute of Technology, Atlanta, GA, United States, ${ }^{2}$ Parker H. \\ Petit Institute for Bioengineering and Bioscience, Georgia Institute of Technology, Atlanta, GA, United States
}

Keywords: metamaterials, acoustics, mechanical, phononic crystal, wave control

\section{Editorial on the Research Topic}

Developments in Acoustic, Phononic, and Mechanical Materials for Wave Control

Wave control at will is of particular interest to the scientific and engineering fields due to its importance in imaging, sensing, and communication. In the past two decades, designing novel structural and artificial composite materials with unprecedented functionalities for wave control has been a hot topic among the research community since the realization of the first acoustic metamaterials with negative effective elastic constant in 2000 (Liu et al., 2000). Acoustic metamaterials are structural materials consisting of deep subwavelength unit cells that achieves different effective dynamic properties based on resonances (Cummer et al., 2016). As an example, the negative effective mass density of the first acoustic metamaterial was realized by operating near the dipolar resonance of the unit cells (Mei et al., 2006). Meanwhile, acoustic metamaterials with negative effective bulk modulus can be achieved by operating near the monopolar resonance of the unit cells

\section{OPEN ACCESS}

Edited and reviewed by: Philip Feng, University of Florida, United States

*Correspondence: Chengzhi Shi chengzhi.shi@gatech.edu

Specialty section:

This article was submitted to Micro- and Nanoelectromechanical

Systems,

a section of the journal Frontiers in Mechanical Engineering

Received: 07 October 2021 Accepted: 25 November 2021 Published: 13 December 2021

Citation:

Shi C (2021) Editorial: Developments in Acoustic, Phononic, and Mechanical Materials for Wave Control. Front. Mech. Eng 7:790830. doi: 10.3389/fmech.2021.790830
(Fang et al., 2006). When designing the structures of the unit cells to attain an overlap of the frequency bands associated with negative density and bulk modulus, the effective refractive index becomes negative (Lee et al., 2010). A metamaterial with negative refractive index can be used as a superlens for super-resolution imaging (i.e., imaging beyond the diffraction limit with deep subwavelength resolution) (Kaina et al., 2015). In addition to resonance-based metamaterials, sonic and phononic crystals with periodic structures were developed to induce frequency bandgaps through Bragg scattering for wave guiding and filtering (Martínez-Sala et al., 1995).

These novel concepts were later extended into mechanical structures and materials for the manipulations of elastic waves, stress, and deformations (Bertoldi et al., 2017). Auxetic metamaterials with negative Poisson's ratio were designed with inverted hexagon patterned structures (Lakes, 1987). Judicious designs of unit cell chirality in deformation have enabled the conversion between compression and twisting (Frenzel et al., 2017). Lattice defects were used to regulate the stress distribution in mechanical lattices (Paulose et al., 2015). The ancient techniques of origami and kirigami have also inspire many new designs of mechanical materials for deformation control to achieve deployable structures, flexible medical stents, and flexible electronic devices (Melancon et al., 2021). Spinning gyros were applied to induce topological effects for robust one-way propagation of elastic wave along the edge of mechanical crystals (Wang et al., 2015). Topological mechanisms were developed to control the propagation of domain walls (Kane and Lubensky, 2014). Nonlinearity of lattice structure was implemented for the realization of nonreciprocal mechanics (Coulais et al., 2017). More recently, these newly obtained mechanical properties have been integrated with the design of acoustic metamaterials to realize a selfadaptive soft acoustic invisibility cloak (Xue and Zhang, 2021). Zhang and Wang discussed the control of rolling elastic waves in anisotropic materials in the article "Boundary Reflections of Rolling Waves in Cubic Anisotropic Material" of this collection. 
While achieving unprecedented material properties is still an exciting direction to pursue, the focus of the research community has started shifting towards the practical applications of the novel functionalities. Acoustic superlens with negative refractive index has the potential to achieve super-resolution imaging, but the fact that the subwavelength images can only be formed near the lens limits its application in biological systems. On the contrary, ultrasound contrast agents including microbubbles and phase-transition nanodroplets provide more practical solutions. Ultrasound localization microscopy with micrometer scale resolution was developed using microbubbles flowing in blood for the visualization of the brain vasculature of a mouse (Errico et al., 2015). The use of phase-transition nanodroplets has the potential to further improve the imaging quality (Luke et al., 2016). However, the presence of the skull prevents the realization of highquality ultrasound brain imaging for large mammals including humankinds due to the strong acoustic impedance mismatch and porosity of the cranial bone. A passive acoustic metamaterial was designed to match the acoustic impedance and reduce ultrasound reflection (Shen et al., 2014), but the ignorance of the porosity induced acoustic attenuation makes the metamaterial impractical in improving the transcranial ultrasound transmission. An active non-Hermitian complementary acoustic metamaterial was proposed to counteract the impedance mismatch and porosity induced loss simultaneously (Craig et al., 2019). In this collection, Craig et al. presented the effect of skull imperfections on the performance of transcranial ultrasound improvement by the use of the non-Hermitian complementary acoustic metamaterial in the article "Non-Hermitian Complementary Acoustic Metamaterials for Imaging Through Skull with Imperfections," showing a significant increase in transcranial ultrasound transmission when properly designed metamaterial is used even for skulls with imperfect geometry and uniformity.

Another major challenge of practical applications of metamaterials is from the nature of their resonance-based designs, limiting the operating frequency band for the control of waves, particularly for low frequency audible range. In the article "Low-Frequency Broadband Acoustic Metasurface

\section{REFERENCES}

Bertoldi, K., Vitelli, V., Christensen, J., and van Hecke, M. (2017). Flexible Mechanical Metamaterials. Nat. Rev. Mater. 2, 17066. doi:10.1038/ natrevmats.2017.66

Coulais, C., Sounas, D., and Alù, A. (2017). Static Non-Reciprocity in Mechanical Metamaterials. Nature 542, 461-464. doi:10.1038/nature21044

Craig, S. R., Welch, P. J., and Shi, C. (2019). Non-Hermitian Complementary Acoustic Metamaterials for Lossy Barriers. Appl. Phys. Lett. 115, 051903. doi:10.1063/1.5110501

Cummer, S. A., Christensen, J., and Alù, A. (2016). Controlling Sound with Acoustic Metamaterials. Nat. Rev. Mater. 1, 16001. doi:10.1038/natrevmats.2016.1

Errico, C., Pierre, J., Pezet, S., Desailly, Y., Lenkei, Z., Couture, O., et al. (2015). Ultrafast Ultrasound Localization Microscopy for Deep Super-Resolution Vascular Imaging. Nature 527, 499-502. doi:10.1038/nature16066
Absorbing Panel" of this collection, Ji et al. coupled multiple types of resonators to extend the operation bandwidth for lowfrequency acoustic absorption. Wang et al. designed a composite perforated partitioned sandwich panel for absorption of lowfrequency sound waves underwater in the article " $A$ Composite Perforated Partitioned Sandwich Panel with Corrugation for Underwater Low-Frequency Sound Absorption" of this collection. Besides acoustic absorption, the control of sound reflection requires prudent designs. In this collection, Qin et al. presented a metasurface consisting of differential phase shifters to achieve broadband control of sound reflection in the article "Acoustic Wave Reflection Control Based on Broadband Differential Phase Shifters."

In addition to the designs of metamaterials, phononic crystals are also commonly used for the control of wave propagation. Reyes et al. applied defects in phononic crystals to realize high quality factor cavity in the article "Optimization of the Spatial Configuration of Local Defects in Phononic Crystals for High $Q$ Cavity" of this collection. Lucklum et al. discussed the use of phononic crystals as a new class of resonant sensors in the article "Phononic Crystal Sensors: A New Class of Resonant Sensors Chances and Challenges for the Determination of Liquid Properties" of this collection.

The development of new materials for the control of acoustic and elastic waves will continue to be an active hot topic among the scientific and engineering research communities. We hope the readers will find this collection to be inspiring for their future research in structural materials and wave propagations.

\section{AUTHOR CONTRIBUTIONS}

The author confirms being the sole contributor of this work and has approved it for publication.

\section{ACKNOWLEDGMENTS}

The author thanks NSF for support under grant number ECCS2102129.

Fang, N., Xi, D., Xu, J., Ambati, M., Srituravanich, W., Sun, C., et al. (2006). Ultrasonic Metamaterials with Negative Modulus. Nat. Mater 5, 452-456. doi:10.1038/nmat1644

Frenzel, T., Kadic, M., and Wegener, M. (2017). Three-dimensional Mechanical Metamaterials with a Twist. Science 358, 1072-1074. doi:10.1126/ science.aao4640

Kaina, N., Lemoult, F., Fink, M., and Lerosey, G. (2015). Negative Refractive Index and Acoustic Superlens from Multiple Scattering in Single Negative Metamaterials. Nature 525, 77-81. doi:10.1038/nature14678

Kane, C. L., and Lubensky, T. C. (2014). Topological Boundary Modes in Isostatic Lattices. Nat. Phys 10, 39-45. doi:10.1038/nphys2835

Lakes, R. (1987). Foam Structures With a Negative Poisson's Ratio. Science 235, 1038-1040. doi:10.1126/science.235.4792.1038

Lee, S. H., Park, C. M., Seo, Y. M., Wang, Z. G., and Kim, C. K. (2010). Composite Acoustic Medium with Simultaneously Negative Density and Modulus. Phys. Rev. Lett. 104, 054301. doi:10.1103/PhysRevLett.104.054301 
Liu, Z., Zhang, X., Mao, Y., Zhu, Y. Y., Yang, Z., Chan, C. T., et al. (2000). Locally Resonant Sonic Materials. Science 289, 1734-1736. doi:10.1126/science.289.5485.1734

Luke, G. P., Hannah, A. S., and Emelianov, S. Y. (2016). Super-Resolution Ultrasound Imaging In Vivo with Transient Laser-Activated Nanodroplets. Nano Lett. 16, 2556-2559. doi:10.1021/acs.nanolett.6b00108

Martínez-Sala, R., Sancho, J., Sánchez, J. V., Gómez, V., Llinares, J., and Meseguer, F. (1995). Sound Attenuation by Sculpture. Nature 378, 241. doi:10.1038/378241a0

Mei, J., Liu, Z., Wen, W., and Sheng, P. (2006). Effective Mass Density of Fluid-Solid Composites. Phys. Rev. Lett. 96, 024301. doi:10.1103/PhysRevLett.96.024301

Melancon, D., Gorissen, B., García-Mora, C. J., Hoberman, C., and Bertoldi, K. (2021). Multistable Inflatable Origami Structures at the Metre Scale. Nature 592, 545-550. doi:10.1038/s41586-021-03407-4

Paulose, J., Chen, B. G.-G., and Vitelli, V. (2015). Topological Modes Bound to Dislocations in Mechanical Metamaterials. Nat. Phys 11, 153-156. doi:10.1038/ nphys 3185

Shen, C., Xu, J., Fang, N., and Jing, Y. (2014). Anisotropic Complementary Acoustic Metamaterial for Canceling Out Aberrating Layers. Phys. Rev. X 4, 041033. doi:10.1103/physrevx.4.041033

Wang, P., Lu, L., and Bertoldi, K. (2015). Topological Phononic Crystals with One-Way Elastic Edge Waves. Phys. Rev. Lett. 115, 104302. doi:10.1103/physrevlett.115.104302
Xue, Y., and Zhang, X. (2021). Self-Adaptive Acoustic Cloak Enabled by Soft Mechanical Metamaterials. Extreme Mech. Lett. 46, 101347. doi:10.1016/j.eml.2021.101347

Conflict of Interest: The author declares that the research was conducted in the absence of any commercial or financial relationships that could be construed as a potential conflict of interest.

Publisher's Note: All claims expressed in this article are solely those of the authors and do not necessarily represent those of their affiliated organizations, or those of the publisher, the editors and the reviewers. Any product that may be evaluated in this article, or claim that may be made by its manufacturer, is not guaranteed or endorsed by the publisher.

Copyright $\odot 2021$ Shi. This is an open-access article distributed under the terms of the Creative Commons Attribution License (CC BY). The use, distribution or reproduction in other forums is permitted, provided the original author(s) and the copyright owner(s) are credited and that the original publication in this journal is cited, in accordance with accepted academic practice. No use, distribution or reproduction is permitted which does not comply with these terms. 